\title{
Prognosis in patients presenting with brain metastasis from an undiagnosed primary tumor
}

\author{
Anthony L. D’Ambrosio, M.D., And Siviero Agazzi, M.D. \\ Department of Neurological Surgery, University of South Florida, Tampa, Florida
}

\begin{abstract}
Object. The aim of this study was to test the validity of the hypothesis that patients in whom brain metastasis is the first indication of an undiagnosed primary tumor have a better chance of survival than similar patients with a known primary lesion.

Methods. Between January 1983 and December 1998, 342 patients with computed tomography-diagnosed brain metastases were treated at a single institution. Information on potential prognostic factors, including primary diagnosis status, was collected retrospectively. Univariate and multivariate analyses were performed to identify prognostic factors related to survival.

Survival was not statistically different between patients with an undiagnosed primary (UDP) lesion and those with a diagnosed primary (DP) tumor ( 6 and 4.5 months, respectively; $p=0.097$ ). In the UDP group (122 patients [36\%]), survival was not affected by the eventual identification of the primary disease ( $\mathrm{p}=$ 0.905). The median survival for the entire population was 5.2 months, with 1-, 2-, and 3-year survival rates of 25,11 , and $4 \%$, respectively. Prognostic factors for the overall population included treatment $(\mathrm{p}<$ $0.0001)$, an age less than 65 years $(\mathrm{p}=0.004)$, discharge status $(\mathrm{p}<0.001)$, absence of systemic metastasis $(\mathrm{p}=0.036)$, and asymptomatic cerebral metastasis $(\mathrm{p}=0.05)$.

Conclusions. Treatment modality was the most significant independent variable affecting survival in patients with brain metastases. Eventual identification of a primary tumor does not affect overall survival; therefore, delaying therapeutic intervention in pursuit of a primary diagnosis may not be appropriate. Data in this study failed to demonstrate a statistically significant difference in survival between patients with UDP and those with DP lesions, on first presenting with brain metastases.
\end{abstract}

KEY WORDS - brain tumor • metastases • prognosis • survival

$\mathrm{B}$ RAIN METASTASES occur in 15 to $40 \%$ of patients with systemic cancer, representing approximately 12 patients per 100,000 of the population per year. ${ }^{5,9,18,25,29,35,39}$ Despite thorough investigation, the primary site of tumor origin will not be detected in up to $15 \%$ of these patients..$^{28}$ Authors of several reports in the literature have described survival outcome in this specific patient population, ${ }^{8,19,20,26,28,34}$ but the data are inconclusive. ${ }^{4} \mathrm{Be}-$ cause the initial clinical signs originate from the brain, this patient population is more likely to present to a neurosurgeon or a neurologist before being referred to an oncologist. ${ }^{32}$ Therefore, it is critical for the neurosurgery community to be familiar with the current prognosis for brain metastases as the first manifestation of a previously undiagnosed primary tumor.

Previously, we have demonstrated that patients presenting with brain metastases as the first manifestation of a

Abbreviations used in this paper: $\mathrm{CI}=$ confidence interval; $\mathrm{CT}=$ computed tomography; DP = diagnosed primary; HR = hazard ratio; KPS = Karnofsky Performance Scale; UDP = undiagnosed primary; WBRT $=$ whole-brain radiotherapy.
UDP tumor are in better general condition at the time of discharge compared with similar patients harboring a DP tumor. ${ }^{1}$ Because the general condition of a patient with brain metastases has been shown to correlate strongly with overall survival, ${ }^{2}$ we decided to test the hypothesis that survival is different between patients with UDP and those with DP lesions presenting with newly diagnosed brain metastases.

\section{Clinical Material and Methods}

The records of all patients with CT-diagnosed brain metastases treated at Centre Hospitalier Universitaire Vaudois, Lausanne, Switzerland, between January 1983 and December 1998 were retrospectively reviewed. Patients with UDP lesions were defined as those in whom brain metastasis was the first sign of a previously UDP tumor. Once classified as having a UDP lesion, patients underwent routine clinical investigations consisting of chest Xray and thoracoabdominal CT scan. Additional studies such as bone scan, mammography, bronchoscopy, or gas- 
TABLE 1

Clinical characteristics on first presentation with brain metastases in 342 patients*

\begin{tabular}{|c|c|c|c|}
\hline \multirow[b]{2}{*}{ Parameter } & \multicolumn{3}{|c|}{ No. of Patients (\%) } \\
\hline & UDP Group & DP Group & Total \\
\hline \multicolumn{4}{|l|}{$\operatorname{sex}$} \\
\hline male & $40(33)$ & $94(43)$ & 134 (39) \\
\hline female & $82(67)$ & $126(57)$ & $208(61)$ \\
\hline \multicolumn{4}{|l|}{ age (yrs) } \\
\hline$<65$ & $83(68)$ & $152(69)$ & $235(69)$ \\
\hline$\geq 65$ & $39(32)$ & $68(31)$ & $107(31)$ \\
\hline \multicolumn{4}{|l|}{ clinical presentation } \\
\hline focal deficit & $78(64)$ & $108(51)$ & $186(56)$ \\
\hline seizure & $22(18)$ & 37 (17) & $59(18)$ \\
\hline intracranial hypertension & $10(8)$ & $23(11)$ & $33(10)$ \\
\hline headache & $7(6)$ & $17(8)$ & $24(7)$ \\
\hline asymptomatic & $4(3)$ & $29(14)$ & $33(10)$ \\
\hline \multicolumn{4}{|l|}{ no. of brain metastases } \\
\hline 1 & $63(52)$ & $118(55)$ & $181(54)$ \\
\hline 2 & $12(10)$ & $28(13)$ & $40(12)$ \\
\hline$>2$ & $46(38)$ & $70(32)$ & $116(34)$ \\
\hline \multicolumn{4}{|l|}{ location of brain metastases } \\
\hline supratentorial & $83(69)$ & $139(66)$ & $222(67)$ \\
\hline infratentorial & $7(6)$ & $22(11)$ & $29(9)$ \\
\hline both & $30(25)$ & $49(23)$ & $79(24)$ \\
\hline \multicolumn{4}{|l|}{ presence of systemic disease } \\
\hline no & $68(61)$ & $98(46)$ & $166(51)$ \\
\hline yes & $44(39)$ & $114(54)$ & $158(49)$ \\
\hline \multicolumn{4}{|l|}{ location of primary tumor } \\
\hline lung & $73(60)$ & $94(43)$ & $167(49)$ \\
\hline breast & $2(2)$ & $34(15)$ & $36(11)$ \\
\hline skin & $3(3)$ & $32(15)$ & $35(10)$ \\
\hline unknown & $32(26)$ & $3(1) \dagger$ & $35(10)$ \\
\hline other & $5(4)$ & $29(13)$ & $34(10)$ \\
\hline colon & $3(3)$ & $18(8)$ & $21(6)$ \\
\hline kidney & $4(3)$ & $10(5)$ & $14(4)$ \\
\hline
\end{tabular}

* Totals below 342 indicate missing values.

$\dagger$ Patients presented with systemic metastases but no isolated primary disease.

\$ Primary locations include bladder, uterus, thyroid, lymphoma, soft tissue, and epidermoid of ear, nose, and throat.

trointestinal endoscopy were performed only when indicated by clinical signs or histological characteristics of the brain lesion.

The number and location of brain metastases were recorded from imaging data and official reports. Other pertinent information, such as clinical symptoms on presentation, treatment modalities implemented, and interval from initial diagnosis of primary tumor to presentation, were recorded. Concerning the primary tumor, its location, histological features, and treatment as well as any accompanying systemic disease at the time of presentation, were recorded. Patients with evidence of extracranial metastases were included in all analyses.

The appropriate treatment for each patient was discussed in a multidisciplinary setting with the participation of neurosurgeons, neurologists, neuropathologists, and oncologists. According to generally accepted guidelines, the treatment plan was significantly influenced by the expected survival period for each patient as reflected by the performance status, number of brain metastases, and the level of control of the primary disease. . $^{211,14,15,27,30,36,39}$ For patients with multiple brain lesions who did not meet surgical criteria, WBRT was administered unless their clinical status was so poor that palliative treatment with steroids alone was advocated.

Follow-up data were collected from hospital charts, outpatient clinic records, and consultation with general practitioners who monitored patients after discharge. Due to the unavailability of KPS scores in all patients, the discharge destination (home as opposed to another hospital or nursing home) was used as an approximation of performance status. Furthermore, discharge to hospice care, whether at home or in a nursing facility, was coded as "discharge to hospital or nursing home." Survival was calculated from the time of the first diagnosis of cerebral metastases to death or to the last date seen alive if the patient was still alive at the time of analysis. Patients lost to follow up were censored once lost.

When comparing continuous and categorical variables between two groups of interest, the chi-square and Kruskal-Wallis tests were used, respectively. ${ }^{3,6}$ For explorative purposes, a multivariate logistic regression ${ }^{4}$ was performed to compare simultaneously all characteristics of interest between patients in the UDP group and those in the DP group. The Kaplan-Meier method ${ }^{17}$ was used to estimate survival experience. A univariate comparison of survival was performed using the log-rank test. ${ }^{31}$ The standard error of survival percentage at any specified point in time was computed according to the Greenwood method. ${ }^{16}$ Univariate and multivariate analyses of overall survival were performed applying the Cox model. ${ }^{7}$ Results of survival comparisons in those with a UDP lesion relative to those with a DP tumor are reported in terms of HRs, their 95\% CI, and corresponding probability values. A significant HR greater than 1 is interpreted as evidence of better survival for those in the DP group and vice versa for a significant HR less than 1 . In all regressions, the variables describing patient characteristics in this report were considered and appropriately coded in terms of binary indicators. To arrive at a final model, backward stepwise selection was used, with variables entering the model if their probability value was less than 5\% and leaving the model if the value was greater than $10 \%$. All reported probability values are two sided. In this study, we observed 306 treatment failures in a group of patients among whom approximately two thirds had a DP lesion and one third a UDP tumor. For a significance level of 5\% $(\mathrm{p}<0.05)$, we were in a position to detect an HR of magnitude 1.45 or larger with $90 \%$ power. All statistical analyses were performed using the Stata computer package (StataCorp, LP). ${ }^{1}$

\section{RESULTS}

Between January 1983 and December 1998, 345 patients presenting with cerebral metastases were admitted to our institution for evaluation. Three patients were excluded from the study because they were referred for stereotactic biopsy and subsequently discharged for further treatment at their primary institutions, leaving 342 patients for analysis. One hundred twenty-two patients (36\%) made up the UDP group, and 220 (64\%) the DP group.

\section{Patient Characteristics}

Patient characteristics on first presentation with brain metastases have been previously reported ${ }^{2}$ and are sum- 
marized in Table 1. Characteristics of patients with UDP and DP lesions were found to be statistically similar with respect to sex, age, treatment, and number and location of brain metastases. A statistically significant difference was demonstrated in the origin of the primary tumor: patients in the UDP group more frequently presented with lung metastases than did those in the DP group (60\% compared with $43 \%$, respectively; $p=0.001$ ). The presence of systemic metastases at the time of presentation, although statistically different between the two groups on univariate analysis $(\mathrm{p}=0.013)$, was not confirmed by multivariate logistic regression. ${ }^{1}$

Resection and WBRT were performed in 107 patients $(31 \%)$, who were in good general condition with a controlled primary tumor. This treatment group included 97 patients with a single metastasis and eight patients with two lesions reachable via the same craniotomy. In two cases, a single symptomatic lesion causing significant neurological deficit was removed in the setting of multiple metastases. Radiosurgery was performed in addition to WBRT in 19 patients (5.6\%). This group included nine patients treated for a single lesion, four treated for two lesions, and six in whom the most symptomatic lesion was treated in the setting of multiple metastases. Whole-brain radiotherapy was administered in 147 patients (43\%), whereas palliative steroid treatment alone was advocated in 69 patients (20\%). These data are presented in Table 2.

Follow up evaluation from presentation until the time of death was performed in 306 patients (89\%), 21 (6\%) were still alive at the time of final data analysis, and $15(4 \%)$ were lost to follow up.

\section{Prognostic Factors}

In the entire population, results of multivariate analysis demonstrated a statistically significant effect on survival for the following prognostic factors: treatment $(\mathrm{p}<$ $0.0001)$, age less than 65 years $(p=0.004)$, discharge home $(\mathrm{p}<0.001)$, absence of systemic metastasis $(\mathrm{p}=$ 0.036 ), and asymptomatic cerebral metastasis (with respect to all other clinical presentation together, $\mathrm{p}=$ $0.05)$. The diagnosis of primary lung disease significantly influenced survival and increased the risk of death with respect to all other sites of origin $(p=0.001)$. Overall median survival of the entire population was 5.2 months, with 1-, 2-, and 3-year survival rates of 25,11 , and $4 \%$, respectively.

\section{Duration of Survival}

The median survival period in patients in the UDP group

TABLE 2

Treatment of 342 patients with brain metastases*

\begin{tabular}{lccc}
\hline \hline \multirow{2}{*}{\multicolumn{1}{c}{ Treatment }} & \multicolumn{3}{c}{ No. of Patients (\%) } \\
\cline { 2 - 4 } & UDP Group & DP Group & Total \\
\hline surgery + WBRT & $46(38)$ & $61(28)$ & $107(31)$ \\
radiosurgery + WBRT & $4(3)$ & $15(7)$ & $19(6)$ \\
WBRT & $45(37)$ & $102(46)$ & $147(43)$ \\
corticosteroids & $27(22)$ & $42(19)$ & $69(20)$ \\
\hline
\end{tabular}

*Totals below 342 indicate missing values.

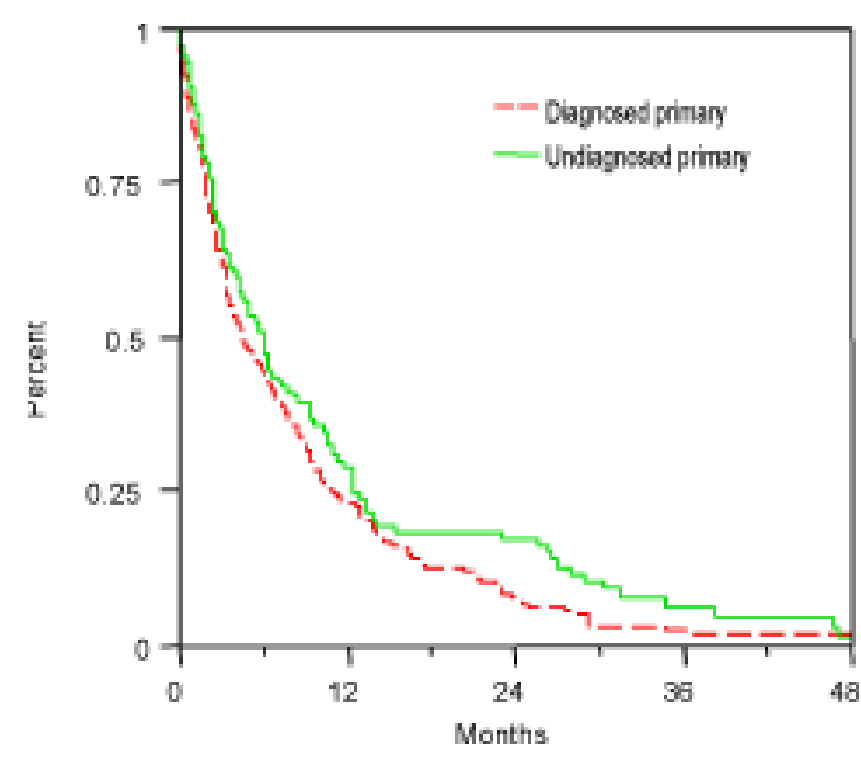

FIG. 1. Graph of Kaplan-Meier curve demonstrating the overall survival rate from the first diagnosis of brain metastases. Overall survival in the UDP group compared with that in the DP group was not statistically different $(\mathrm{p}=0.097)$.

was 6 months, with 1-, 2-, and 3-year survival rates of 29, 17 , and $6 \%$, respectively. The median survival in patients in the DP group was 4.5 months, with 1-, 2-, and 3-year survival rates of 23,8 , and $3 \%$, respectively. Univariate analysis data failed to demonstrate a significant difference between the survival experience of patients in the UDP group and those in the DP group ( $\mathrm{HR}=1.22,95 \% \mathrm{CI}$ $0.96-1.55, \mathrm{p}=0.097)$. Multivariate analysis data confirmed this lack of difference in survival $(\mathrm{HR}=1.18,95 \%$ CI $0.91-1.54, \mathrm{p}=0.213$ ). Survival comparisons for the UDP and DP groups are illustrated in Fig. 1 and summarized in Table 3.

Among patients in the UDP group, survival did not differ according to whether the primary tumor was eventually found or remained unknown $(\mathrm{p}=0.905$; Fig. 2 and Table 3). A trend toward longer survival was noted when systemic disease was absent, although this difference did not reach statistical significance $(p=0.03)$. Among patients in the DP group, the duration of time between the diagnosis of the primary tumor and that of brain metastases did not significantly influence survival. This result was confirmed, in the presence of other factors, by a multivariate regression for survival in this subgroup of patients $(p=0.153)$. When analysis was restricted to only those with UDP lesions and favorable prognostic indicators (32 patients; discharge home, absence of extracranial metastasis, and treatment with surgery plus WBRT or radiosurgery plus WBRT), a median survival of 13.7 months was observed (Fig. 3). The leading cause of death in the majority of patients was systemic disease progression.

\section{Discussion}

Data in this study failed to demonstrate a statistically significant difference in survival between patients in whom brain metastases were the first indication of a previously undiagnosed primary tumor and those presenting 
TABLE 3

Results of univariate analysis*

\begin{tabular}{|c|c|c|c|c|c|c|}
\hline \multirow[b]{2}{*}{ Parameter } & \multirow[b]{2}{*}{ No. of Patients } & \multicolumn{5}{|c|}{ Survival } \\
\hline & & Median (mos) & $1-$ Year $(\%)$ & 2 -Year $(\%)$ & 3 -Year $(\%)$ & $\mathrm{p}$ Value $\dagger$ \\
\hline overall & 342 & 5.2 & 25 & 11 & 4 & \\
\hline \multicolumn{7}{|l|}{ primary status } \\
\hline DP group & 220 & 4.5 & 23 & 8 & 3 & 0.097 \\
\hline UDP group & 122 & 6 & 29 & 17 & 6 & \\
\hline \multicolumn{7}{|c|}{ primary eventually found in UDP group $\$$} \\
\hline no & 32 & 3.2 & 39 & 24 & 8 & 0.905 \\
\hline yes & 90 & 6.2 & 25 & 15 & 6 & \\
\hline \multicolumn{7}{|c|}{ presence of systemic disease in UDP group } \\
\hline present & 44 & 4.2 & 15 & 8 & 9 & 0.03 \\
\hline absent & 67 & 9.8 & 41 & 23 & 6 & \\
\hline \multicolumn{7}{|c|}{$\begin{array}{l}\text { time from primary tumor diagnosis to } \\
\text { presence of brain metastasis in DP group }\end{array}$} \\
\hline$<6$ months & 58 & 5.7 & 23 & 7 & $\mathrm{NE}$ & 0.061 \\
\hline $6-36$ months & 105 & 3.4 & 16 & 5 & 4 & \\
\hline$>36$ months & 54 & 7.9 & 36 & 10 & 2 & \\
\hline \multicolumn{7}{|l|}{ treatment } \\
\hline surgery + WBRT & 107 & 12.6 & 53 & 24 & 11 & $<0.0001$ \\
\hline radiosurgery + WBRT & 19 & 11.4 & 47 & 12 & $\mathrm{NE}$ & \\
\hline WBRT & 147 & 3.5 & 12 & 4 & $\mathrm{NE}$ & \\
\hline corticosteroids & 69 & 1.4 & 3 & 1 & $\mathrm{NE}$ & \\
\hline
\end{tabular}

$* \mathrm{NE}=$ not estimable.

$\dagger$ Overall log-rank test.

\$ Values less than 342 indicate missing values and/or subgroup analysis.

with brain metastases from a known primary lesion. Furthermore, eventual identification of the primary tumor did not affect overall survival. Favorable prognostic factors in the entire population of patients with newly diagnosed brain metastases included treatment modality, age less than 65 years, discharge home, absence of systemic disease, and asymptomatic presentation. The diagnosis of primary lung cancer significantly influenced survival and

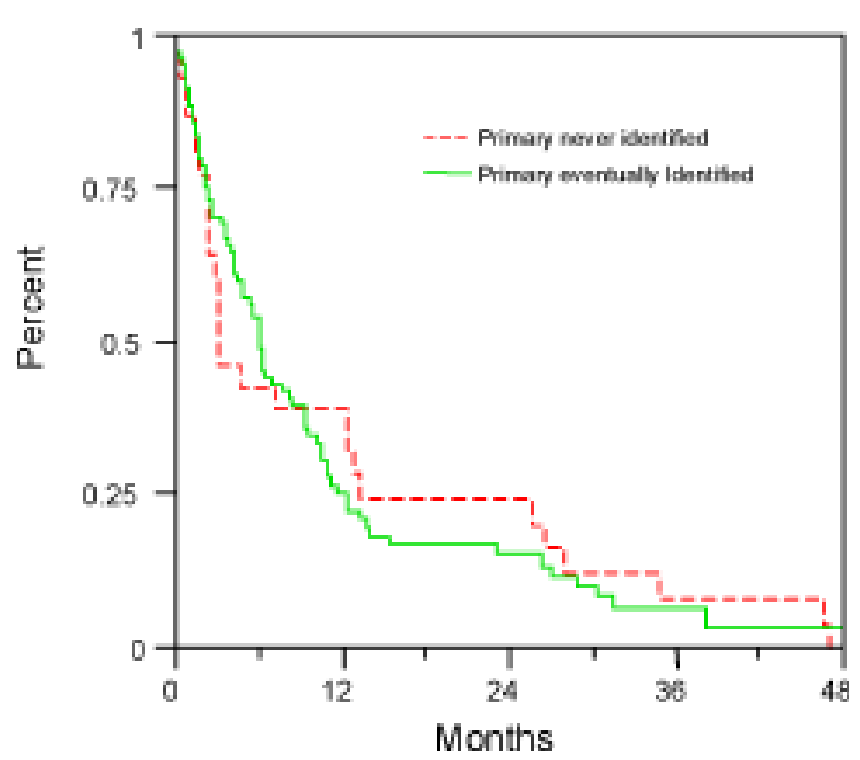

FIG. 2. Graph of Kaplan-Meier curve revealing the overall survival rate in patients with UDP tumors, according to whether or not a primary tumor was eventually identified. Overall survival based on the eventual identification of a primary lesion was not statistically different $(\mathrm{p}=0.905)$. increased the risk of death with respect to all other primary sites.

A clear understanding of the definition of a patient with a UDP lesion is critical to interpretation and a comparison of these results with those in other similar series. Several authors have grouped together patients whose brain metastases were the first symptom of a tumor discovered at diagnosis and patients whose primary tumor remained undetected after a thorough investigation. ${ }^{8,10,24,34,38}$ Other authors have required extensive clinical investigations over a specific period of time before a patient can be classified as having an undiagnosed primary lesion. ${ }^{4,22,26}$ Still other investigators do not clearly establish the period of time after which a patient is defined as having an undiagnosed primary lesion, ${ }^{8,12,24,33,34}$ thus making results across studies difficult to compare. ${ }^{4}$ In the current study, patients in the UDP group were classified in terms we believe to be clinically relevant to the practicing neurosurgeon and neurologist: patients were assigned to the UDP group if they first presented with brain metastases due to a previously undiagnosed primary tumor. This classification takes into account the observation that patients fitting this description are more likely to present first to a neurosurgeon or neurologist rather than an oncologist because of clinical symptomatology originating from the brain. ${ }^{32}$ This definition also takes into consideration previous findings that a significant number of patients with UDP lesions either will have primary lung disease or remain undiagnosed despite expensive and time-consuming clinical investigations. ${ }^{1,24,33,38}$

Applying this classification algorithm, we studied data from 342 cases to determine whether patients in whom brain metastases were the first presentation of a previously UDP tumor have a better chance of survival than similar patients with a known primary lesion. Despite the fact 


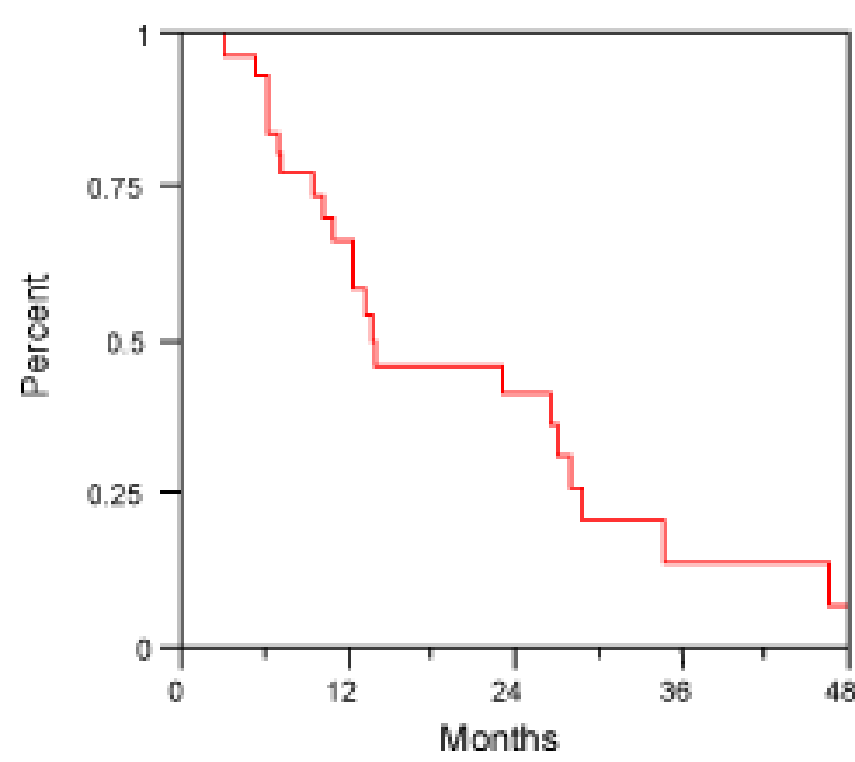

FIG. 3. Graph of Kaplan-Meier curve showing overall survival rate in patients with UDP tumors and favorable prognostic factors at the time of first diagnosis. When analysis was restricted to those with UDP tumors and favorable prognostic indicators (discharge home, absence of extracranial metastasis, and treatment with surgery + WBRT or radiosurgery + WBRT; 32 patients), a median survival of 13.7 months was observed.

that patients with UDP lesions had a higher overall functional status and were more likely to be discharged directly home after treatment, ${ }^{1}$ a statistically significant difference in survival was not observed. These results support the findings of a recent retrospective series in which the authors studied 916 patients who had been treated at a single institution for newly diagnosed brain metastases. ${ }^{4}$ In this report, 47 patients $(5.1 \%)$ presented with cancer of previously unknown origin, whereas 869 patients $(94.9 \%)$ presented with a known primary diagnosis. The median survival in patients with disease of previously unknown origin was 4.8 months and showed no significant difference compared with the overall survival of 3.4 months for patients with brain metastases and previously diagnosed primary disease. Actuarial survival rates for those with cerebral metastases and previously undiagnosed primary disease were 26 and $5 \%$ at 1 and 2 years, respectively, which are rates that compare favorably with findings in the current study.

In a retrospective study by Nguyen et al., ${ }^{26}$ a significantly higher median overall survival rate of 13.4 months was demonstrated in a select group of patients with brain metastases in whom the primary tumor remained undetected despite extensive clinical evaluation. By the authors' definition, patients in whom either the primary site was found or extracranial metastases were discovered within 2 months of the brain metastases were completely excluded from analysis. The authors asserted that the more favorable prognosis in those with a UDP lesion was most likely due to the absence of extracranial disease and that the primary occult tumor posed no immediate threat to survival. Similarly, a median survival of 15 months was demonstrated in a select group of 15 high-functioning patients (median KPS Score 90) with brain metastases from an unknown origin after treatment with radiosurgery. ${ }^{22}$ In the present study, patients were included if no primary lesion was detected in the first 3 months after diagnosis, despite an extensive evaluation including chest radiography; CT scan of the chest, abdomen, and pelvis; and gastrointestinal endoscopy. Poor prognostic factors included the location of metastases in the brainstem and the presence of extracranial disease. For comparison, we performed a subgroup analysis restricted to patients with UDP lesions and favorable prognostic factors. In this population, a median survival of 13.7 months was observed, suggesting that longer survival can be seen in a select subgroup of patients with UDP lesions.

In the current study, the eventual identification of a primary lesion in patients with UDP lesions did not affect overall survival. This result supports recent findings by Ruda et al., ${ }^{33}$ who prospectively studied 33 patients with brain metastases from an unknown primary site that remained unknown after initial diagnostic exploration. Gross-total resection and WBRT were performed in 26 patients (79\%), and the median overall survival was 10 months. After treatment, the primary diagnosis remained unknown in $18 \%$ of patients (six of 33) and was found to be lung cancer in the remaining cases. Moreover, eventual primary diagnosis did not significantly affect survival. Therefore, the authors concluded that a costly and extensive evaluation for an undetected primary tumor may not be appropriate during follow-up evaluation until more effective cancer therapy is available. .4,33,38 $^{2}$

Due to the retrospective nature of the current study, the KPS score was not available in all patients. As a surrogate measure, the discharge destination (home as opposed to another hospital or nursing home) was used as an approximation of performance status. To minimize error introduced by this methodology, discharge to hospice care, whether at home or in a nursing facility, was coded as "discharge to hospital or nursing home." Results of the current study should be interpreted with this methodology in mind.

\section{Conclusions}

Data regarding the prognosis of brain metastases from an undiagnosed primary tumor are scattered throughout the literature. ${ }^{8,13,19,21-24,26,28,34,37}$ Given that projected survival heavily influences treatment selection, it is important to determine whether brain metastases in patients with UDP lesions have a different prognosis from cerebral metastases in those with DP lesions.

Our results suggest that once a patient presents with brain metastases, overall prognosis is not affected by previous knowledge of a primary tumor. Furthermore, delaying treatment intervention in pursuit of a primary diagnosis may not be appropriate as the eventual identification of a primary lesion has not been shown to influence prognosis. Survival beyond 1 year can occur in patients with more favorable prognostic factors at the first diagnosis of brain metastases; however, when a practical and clinically applicable definition of a patient with an "undiagnosed primary lesion" is used, the prognosis in this population is no different from the prognosis in the overall population with brain metastases. 


\section{References}

1. Agazzi S, Pampallona S, Pica A, Vernet O, Regli L, Porchet F, et al: The origin of brain metastases in patients with an undiagnosed primary tumor. Acta Neurochir (Wien) 146:153-157, 2004

2. Agboola O, Benoit B, Cross P, Da Silva V, Esche B, Lesiuk H, et al: Prognostic factors derived from recursive partition analysis (RPA) of Radiation Therapy Oncology Group (RTOG) brain metastases trials applied to surgically resected and irradiated brain metastatic cases. Int J Radiat Oncol Biol Phys 42: 155-159, 1998

3. Armitage P, Berry G: Statistical Methods in Medical Research, ed 2. Oxford: Blackwell Science, 1987

4. Bartelt S, Lutterbach J: Brain metastases in patients with cancer of unknown primary. J Neurooncol 64:249-253, 2003

5. Cairncross JG, Kim JH, Posner JB: Radiation therapy for brain metastasis. Ann Neurol 7:529-541, 1980

6. Conover WJ: Practical Nonparametric Statistics. New York: John Wiley \& Sons, 1971

7. Cox DR: Regression models and life tables. J R Stat Soc B 34: 187-220, 1972

8. Debevec M: Management of patients with brain metastases of unknown origin. Neoplasma 37:601-606, 1990

9. Delattre JY, Krol G, Thaler HT, Posner JB: Distribution of brain metastases. Arch Neurol 45:741-744, 1988

10. Dhopesh VP, Yagnik PM: Brain metastasis: analysis of patients without known cancer. South Med J 78:171-172, 1985

11. Diener-West M, Dobbins TW, Phillips TL, Nelson DF: Identification of an optimal subgroup for treatment evaluation of patients with brain metastases using RTOG study 7916. Int J Radiat Oncol Biol Phys 16:669-673, 1989

12. Eapen L, Vachet M, Catton G, Danjoux C, McDermot R, Nair $\mathrm{B}$, et al: Brain metastases with an unknown primary: a clinical perspective. J Neurooncol 6:31-35, 1988

13. Ebels E, van der Meulen JD: Cerebral metastasis without known primary tumor: a retrospective study. Clin Neurol Neurosurg 80:195-197, 1978

14. Fuller BG, Kaplan ID, Adler J, Cox RS, Bagshaw MA: Stereotactic radiosurgery for brain metastases: the importance of adjuvant whole brain irradiation. Int J Radiat Oncol Biol Phys 23:413-418, 1992

15. Gaspar L, Scott C, Rotman M, Asbell S, Phillips T, Wasserman $\mathrm{T}$, et al: Recursive partitioning analysis (RPA) of prognostic factors in three Radiation Therapy Oncology Group (RTOG) brain metastases trials. Int J Radiat Oncol Biol Phys 37: 745-751, 1997

16. Greenwood M: The natural duration of cancer, in Ministry of Health: Reports on Public Health and Medical Subjects, No. 33. London: His Majesty's Stationery Office, 1926

17. Kaplan EL, Meier P: Nonparametric estimation from incomplete observations. J Am Stat Assoc 53:457-481, 1958

18. Kehrli P: [Epidemiology of brain metastases.] Neurochirurgie 45:357-363, 1999 (Fr)

19. Khansur T, Routh A, Hickman B: Brain metastases from unknown primary site. J Miss State Med Assoc 38:238-242, 1997

20. Lagerwaard FJ, Levendag PC, Nowak PJ, Eijkenboom WM, Hanssens PE, Schmitz PI: Identification of prognostic factors in patients with brain metastases: a review of 1292 patients. Int J Radiat Oncol Biol Phys 43:795-803, 1999

21. Le Chevalier T, Smith FP, Caille P, Constans JP, Rouesse JG: Sites of primary malignancies in patients presenting with cerebral metastases. A review of 120 cases. Cancer 56:880-882, 1985

22. Maesawa S, Kondziolka D, Thompson TP, Flickinger JC, Dade
L: Brain metastases in patients with no known primary tumor. Cancer 89:1095-1101, 2000

23. Mavrakis AN, Halpern EF, Barker FG II, Gonzalez RG, Henson JW: Diagnostic evaluation of patients with a brain mass as the presenting manifestation of cancer. Neurology 65:908-911, 2005

24. Merchut MP: Brain metastases from undiagnosed systemic neoplasms. Arch Intern Med 149:1076-1080, 1989

25. Mintz AH, Kestle J, Rathbone MP, Gaspar L, Hugenholtz H, Fisher B, et al: A randomized trial to assess the efficacy of surgery in addition to radiotherapy in patients with a single cerebral metastasis. Cancer 78:1470-1476, 1996

26. Nguyen LN, Maor MH, Oswald MJ: Brain metastases as the only manifestation of an undetected primary tumor. Cancer 83:2181-2184, 1998

27. Noordijk EM, Vecht CJ, Haaxma-Reiche H, Padberg GW, Voormolen JHC, Hoekstra FH, et al: The choice of treatment of single brain metastasis should be based on extracranial tumor activity and age. Int J Radiat Oncol Biol Phys 29:711-717, 1994

28. Nussbaum ES, Djalilian HR, Cho KH, Hall WA: Brain metastases. Histology, multiplicity, surgery and survival. Cancer 78:1781-1788, 1996

29. Patchell RA: Brain metastases. Neurol Clin 9:817-824, 1991

30. Patchell RA, Tibbs PA, Walsh JW, Dempsey RJ, Maruyama Y, Kryscio RJ, et al: A randomized trial of surgery in the treatment of single metastases to the brain. N Engl J Med 322:494-500, 1990

31. Peto R, Pike MC, Armitage P, Breslow NE, Cox DR, Howard $\mathrm{SV}$, et al: Design and analysis of randomized clinical trials requiring prolonged observation of each patient. II. Analysis and examples. Br J Cancer 35:1-39, 1977

32. Polyzoidis KS, Miliaras G, Pavlidis N: Brain metastasis of unknown primary: a diagnostic and therapeutic dilemma. Cancer Treat Rev 31:247-255, 2005

33. Ruda R, Borgognone M, Benech F, Vasario E, Soffietti R: Brain metastases from unknown primary tumor: a prospective study. J Neurol 248:394-398, 2001

34. Salvati M, Cervoni L, Raco A: Single brain metastases from unknown primary malignancies in CT-era. J Neurooncol 23:75-80, 1995

35. Sheehan J, Niranjan A, Flickinger JC, Kondziolka D, Lunsford LD: The expanding role of neurosurgeons in the management of brain metastases. Surg Neurol 62:32-41, 2004

36. Smalley SR, Schray MF, Laws ER Jr, O'Fallon JR: Adjuvant radiation therapy after surgical resection of solitary brain metastasis: association with pattern of failure and survival. Int J Radiat Oncol Biol Phys 13:1611-1616, 1987

37. Trillet V, Catajar JF, Croisile B, Turjman F, Aimard G, Bourrat $\mathrm{C}$, et al: Cerebral metastases as first symptom of bronchogenic carcinoma. A prospective study of 37 cases. Cancer 67:2935-2940, 1991

38. Van de Pol M, van Aalst VC, Wilmink JT, Twijnstra A: Brain metastases from an unknown primary tumor: which diagnostic procedures are indicated? J Neurol Neurosurg Psychiatry 61: 321-323, 1996

39. Vecht CJ: Clinical management of brain metastasis. J Neurol 245:127-131, 1998

Manuscript submitted December 21, 2006.

Accepted in final form January 26, 2007.

Address reprint requests to: Anthony L. D’Ambrosio, M.D., Department of Neurological Surgery, University of South Florida, Harbourside Medical Tower, 4 Columbia Drive, Suite 730, Tampa, FL 33606. email: djtonyd16@yahoo.com. 